\title{
Analysis of TDP-43 and its binding partners in neurodegenerative diseases
}

\author{
Wejdan Kattuah ${ }^{1 *}$, Claire Troakes ${ }^{1}$, Tibor Hortobagyi ${ }^{2}$, Boris Rogelj ${ }^{3}$, Christopher Shaw ${ }^{1}$ \\ From Molecular Neurodegeneration: Basic biology and disease pathways \\ Cannes, France. 10-12 September 2013
}

\section{Background}

Transactive DNA binding protein (TDP-43) is the major component of the ubiquitin-positive protein aggregates seen in $~ 90 \%$ of Amyotrophic Lateral Sclerosis (ALS) and $60 \%$ of Frontotemporal Lobar Degeneration (FTLD) cases [1]. We have previously shown that mutations in the gene encoding TDP-43 are causally linked to familial ALS+/-FTLD [2] TDP-43 belongs to the heterogeneous nuclear ribonucleoprotein (hnRNP) family of proteins that are involved in the regulation of RNA transcription, splicing, transport and translation [3]. There are a large number of hnRNPs, many of which have overlapping functions and often act cooperatively in RNA processing. Here we sought to determine whether TDP-43 aggregates contain other hnRNPs that might contribute to the neurodegenerative process.

\section{Materials and methods}

Immunohistochemistry for 14 hnRNPs predicted to associate with TDP-43 were examined in brain and spinal cord tissues from 20 FTLD-TDP and ALS cases. Co-localization with TDP-43 was examined using doublelabelling immunoflouresence. Expression of selected hnRNPs was then examined in other neurodegenerative cases and controls to determine the specificity of any changes observed.

\section{Results}

One hnRNP demonstrated a striking accumulation within dystrophic neurites and cytoplasmic inclusions in the frontal cortex of FTLD-TDP cases. The hnRNP inclusions were not detected in other neurodegenerative cases with mutations of MAPT, FUS, SOD1 and C9ORF72. This particular hnRNP was found to co-localize with $\sim 85 \%$ of

'Department of Clinical Neuroscience, Institute of Psychiatry, King's College London, London, UK

Full list of author information is available at the end of the article
TDP-43 inclusions and $\sim 67 \%$ of ubiquitin incluions, largely in the frontal cortex and hippocampus of FTLDTDP cases. Interestingly, the inclusions were not seen in FTLD-TDP cases with C9ORF72 mutation.

\section{Conclusions}

We have identified one hnRNP that is abundant within the inclusions seen in FTLD-TDP cases. The hnRNP is seen to colocalize with TDP-43 in the majority of cytoplasmic inclusions in FTLD-TDP but not other neurodegenerative disorders. The mechanistic implications of this interaction with TDP-43 and the contribution of its sequestration into inclusions towards neurodegeneration requires further investigation.

\section{Authors' details}

'Department of Clinical Neuroscience, Institute of Psychiatry, King's College London, London, UK. ${ }^{2}$ Department of Neuropathology, Institute of

Pathology, Medical and Health Science Centre, University of Debrecen., Debrecen, Hungary. ${ }^{3}$ Department of Biotechnology B3, Jozef Stefan Institute, Jamova 39, Ljubljana, Slovenia.

Published: 13 September 2013

\section{References}

1. Neumann M, Sampathu D, Kwong L, Truax A, Micsenyi M, Chou T, Bruce J, Schuck T, Grossman M, Clark C, McCluskey L, Miller B, Masliah E, Mackenzie I, Feldman H, Kretzschmar H, Trojanowski J: Ubiquitinated TDP43 in Frontotemporal Lobar Degeneration and Amyotrophic Lateral Sclerosis. Science 2006, 10:130-133.

2. Sreedharan J, Blair IP, Tripathi VB, Hu X, Vance C, Rogelj B, Ackerley S, Durnall JC, Williams KL, Buratti E, Baralle F, de Belleroche J, Mitchell JD, Leigh PN, Al-Chalabi A, Miller CC, Nicholson G, Shaw CE: TDP-43 Mutations in Familial and Sporadic ALS. Science 2008, 319:1668-1672.

3. Buratti E, Baralle FE: Multiple roles of TDP-43 in gene expression, splicing regulation, and human disease. Front Biosci 2008, 13:867-878.

\section{doi:10.1186/1750-1326-8-S1-P23}

Cite this article as: Kattuah et al:: Analysis of TDP-43 and its binding partners in neurodegenerative diseases. Molecular Neurodegeneration 2013 8(Suppl 1):P23. 